Gut, 1982, 23, 371-375

\title{
Palliative treatment of obstructive jaundice by transpapillary introduction of large bore bile duct endoprosthesis
}

\author{
Experience in 45 patients
}

\author{
K HUIBREGTSE and G N TYTGAT \\ From the University of Amsterdam, Department of Medicine, Division of Gastroenterology, Wilhelmina \\ Gasthuis, Amsterdam, The Netherlands
}

SUMMARY The endoscopic techniques for introducing a large calibre $(3.2 \mathrm{~mm})$ biliary endoprosthesis to relieve jaundice are described. In 45 patients such an endoprosthesis was successfully introduced. The median survival time in 18 patients was 41 days (range three to 187). Twenty-six patients are still alive after a median of 28 days (range seven to 244). One patient had a surgical bypass after 24 days. Short-term complications were cholangitis and fever in 11 patients. Late complications were recurrent jaundice in four, upward migration of the endoprosthesis in two, and clogging of the endoprosthesis in one patient. The risk of cholangitis, which used to be a major problem with transpapillary introduced prostheses of smaller calibre, seems definitely diminished by using one with a large calibre.

Endoscopic retrograde cholangiopancreatography (ERCP) is an important diagnostic procedure to disclose the anatomy and nature of bile duct obstructions. ${ }^{12}$ In addition, decompression of the obstructed biliary system can be achieved by introduction of a nasobiliary suction catheter ${ }^{3}{ }^{4}$ or a bile duct endoprosthesis. 56

In 1979 Soehendra $e t a l^{5}$ described a method for transpapillary introduction of a biliary endoprosthesis. With a slightly modified technique we successfully introduced 21 such biliary endoprostheses (outer diameter: $2 \cdot 1 \mathrm{~mm}$, inner diameter: $1.6 \mathrm{~mm})$. Many of these patients developed fever and severe cholangitis despite prophylactic antibiotics. We ascribed this complication to the slow bile flow through and the easy clogging of such small calibre endoprostheses and developed a method to introduce a large calibre biliary endoprosthesis via the papillary route. ${ }^{78}$

This communication describes the technique and the results in 45 patients.

Received for publication 19 October 1981

\section{Methods}

TECHNIQUE

An ERCP was first performed to obtain information about the biliary and pancreatic anatomy. An endoscopic papillotomy was carried out, which facilitates the introduction of the various catheters. A 4 metre long balloon catheter (Fogarty $5 \mathrm{~F}$ ) was introduced and pushed through and beyond the bile duct stenosis. The balloon was inflated above the stenosis and remained inflated during the whole procedure. Thereafter the endoscope was removed. Over the balloon catheter as a guide the GIF IT with a biopsy channel of $3.8 \mathrm{~mm}$ was introduced. The endoprosthesis (Fig. 1) was pushed over the balloon catheter through the biopsy channel by a long pusher catheter of the same diameter. The tip of this forward viewing endoscope was pulled to the papillotomy opening by pulling the balloon catheter. The endoscope with an acutely bent tip was held in a fixed position, while the endoprosthesis was pushed through the bile duct obstruction. After successful positioning of the endoprosthesis the balloon was desufflated and the balloon catheter, endoscope, and pusher tube were removed, leaving the 


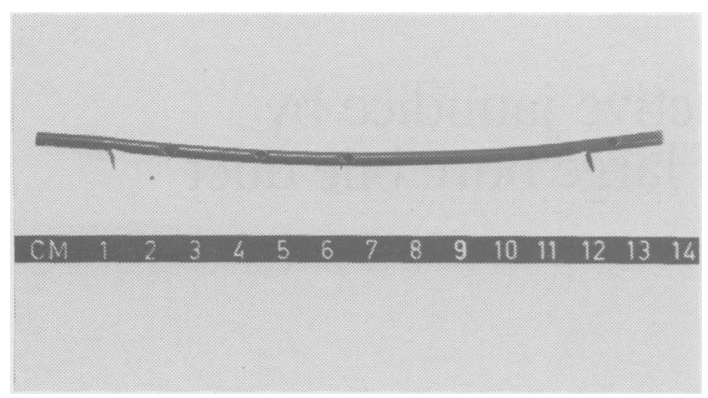

Fig. 1 Biliary endoprosthesis, polyethylene tubing, 12-15 $\mathrm{cm}$ long, many side holes, outer diameter $3.2 \mathrm{~mm}$, inner diameter $2.4 \mathrm{~mm}$ (Surgimed, Denmark).

prosthesis behind. This method was used in 13 patients.

In 12 patients, instead of the GIF IT, we used a specially constructed introducer, made from an old duodenoscope. Out of this duodenoscope the fibre bundles and the biopsy channel were removed and exchanged for a $3.8 \mathrm{~mm}$ instrumentation channel. This introducer was inserted over the balloon catheter and positioned in front of the papilla exactly like a duodenoscope for ERCP under constant fluoroscopic control. Through the instrumentation channel the endoprosthesis was pushed into position over the balloon catheter. In six patients a modified Fujinon duodenoscope was used. Along this duodenoscope a $4 \mathrm{~mm}$ internal diameter Teflon tube was loosely connected. At the tip of the endoscope the tube entered the duodenoscope where the biopsy channel ended. The same bridge was used to bend the cannulation catheter and the catheters leaving the outside instrumentation channel.

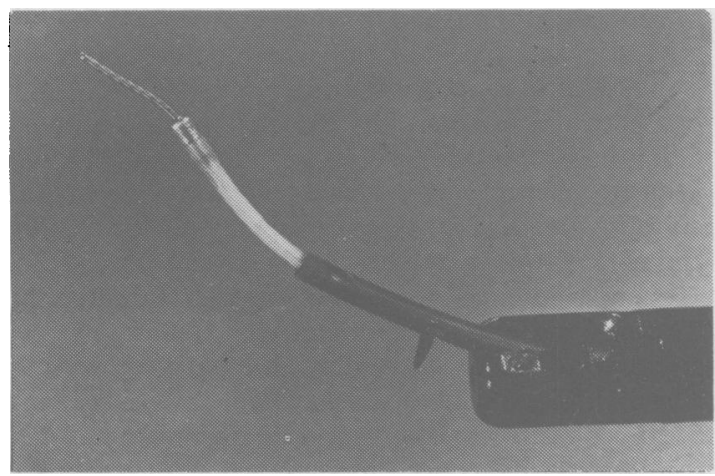

Fig. 2 Prototype Olympus duodenoscope with a $3.7 \mathrm{~mm}$ instrumentation channel. Guide wire, catheter with metal tip, and endoprosthesis leaving this large channel.

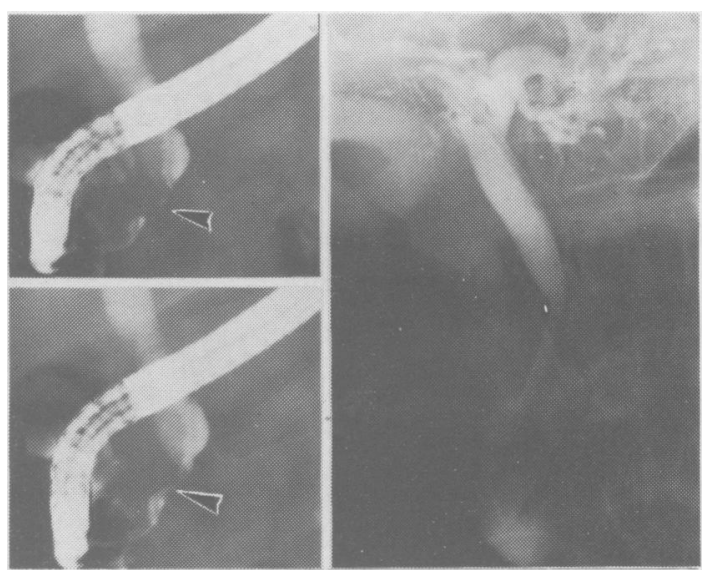

Fig. 3 Left: distal common duct stenosis (arrow), because of histologically proven pancreas carcinoma. Right: X-ray contrast drops through the endoprosthesis in the duodenum.

In 14 patients a new Olympus prototype duodenoscope (Fig. 2) with a $3.7 \mathrm{~mm}$ biopsy channel was used. With this endoscope it is possible to perform the ERCP, the papillotomy, and the introduction of the endoprosthesis in one procedure. After the papillotomy a $2 \mathrm{~m}$ long, $2 \cdot 1 \mathrm{~mm}$ diameter catheter, carrying a $2.5 \mathrm{~m}$ long flexible guide wire was manoeuvred through the bile duct stenosis. Over the catheter the endoprosthesis was pushed into position. Alternatively, the long balloon catheter mentioned above can be used.

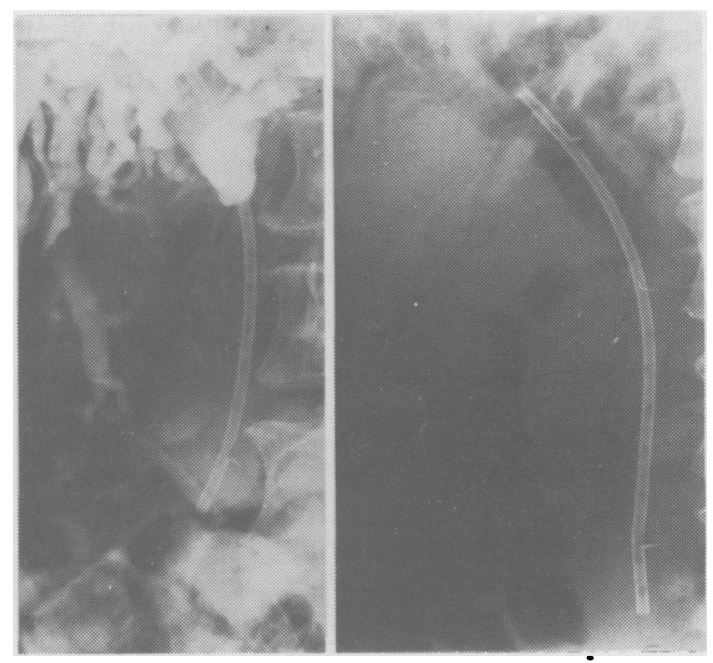

Fig. 4 Left: biliary endoprosthesis inserted through malignant bile duct stricture. Right: four weeks later still an air cholangiography is present. 
An endoprosthesis with one barbed hook was used in 34 patients. An endoprosthesis with two barbed hooks was inserted in 11 patients (Figs 3, 4).

Prophylactic antibiotics (gentamycin) were given immediately after the diagnostic ERCP to the first 21 patients. In five other patients gentamycin was administered because the attempt failed to introduce an endoprosthesis immediately after the diagnostic ERCP.

\section{PATIENTS}

Between August 1980 and June 1981 insertion of a large bore biliary endoprosthesis was attempted in 54 patients ( 28 women, 26 men) mean age 68.5 years (range 42-92). In 51 patients the biliary obstruction was due to malignancy, in two patients to a postoperative stricture in the porta hepatis and in one patient to sclerosing cholangitis. In six patients the obstruction was localised at the bifurcation, in eight patients at the mid common duct, and in 40 patients at the distal common duct. A pancreatic, biliary, and papillary carcinoma was present in respectively 33,14 , and two patients. Two patients had secondaries of respectively a lung- and a gastric carcinoma.

Most patients were considered at high risk for any method of surgical treatment, because of age or concomitant diseases, such as cardiopulmonary insufficiency, diabetes, hypertension, and general debility. Furthermore, in 14 patients surgical treatment was considered impossible because of the localisation of the stenosis and the extent of malignancy in the right upper quadrant. In two patients a surgical bypass procedure had been unsuccessful. In three patients the percutaneous positioning of an endoprosthesis had failed. In one patient the endoprosthesis was inserted as a preoperative drainage procedure.

\section{Results}

In 45 patients a large bore endoprosthesis was successfully introduced. In three patients, early in our experience we were unable to push the endoprosthesis through the stenosis. In two patients the stenosis could not be passed by any type of catheter or guide wire. In four patients with an obstruction near the papilla it was impossible to perform a papillotomy and to pass the stenosis. In 34 patients the endoprosthesis was inserted immediately after the diagnostic ERCP. In 11 patients the first attempt to introduce an endoprosthesis failed, but a second attempt the next day was successful. In 43 patients a decline of the bilirubin level was observed. Itching, when present, disappeared within a few days in all patients. In two patients with multiple liver metastases, no decline of bilirubin levels was seen despite the endoscopically controlled patency of the endoprosthesis.

Bilirubin levels declined to normal or nearly normal levels in 25 patients after an average time interval of about one month (Table). Eighteen patients died after a median time of 41 days (range three to 187 days). Four patients died the first week after insertion of the endoprosthesis because of liver insufficiency, despite declining bilirubin levels in two of them.

Nine patients died without jaundice. Five patients had recurrent jaundice shortly before death. In two of them necropsy was performed and a patent endoprosthesis with tumour growth above the endoprosthesis was found. In three of them necropsy was not allowed. One patient was treated with a surgical bypass 24 days after insertion of the endoprosthesis.

Twenty-six patients are still alive with a wellfunctioning endoprosthesis after a median time interval of 28 days (range seven to 244 days).

\section{Complications}

Fever and chills occurred in 11 patients the day after

Table Bilirubin levels and survival time of 25 patients, followed up for at least 30 days

\begin{tabular}{|c|c|c|c|c|}
\hline \multirow[b]{2}{*}{$\begin{array}{l}\text { Patient } \\
\text { (no) }\end{array}$} & \multicolumn{2}{|c|}{$\begin{array}{l}\text { Bilirubin levels } \\
(<17 \mu \mathrm{mol} / \mathrm{l})\end{array}$} & \multirow[b]{2}{*}{ Alive } & \multirow[b]{2}{*}{$\begin{array}{l}\text { Survival } \\
\text { (days) }\end{array}$} \\
\hline & $\begin{array}{l}\text { Time of } \\
\text { insertion }\end{array}$ & $\begin{array}{l}\text { After } \\
1 \text { month }\end{array}$ & & \\
\hline 1 & 275 & 25 & - & 34 \\
\hline 2 & 261 & 12 & - & 155 \\
\hline 3 & 230 & 17 & - & 125 \\
\hline 4 & 299 & 28 & + & 244 \\
\hline 5 & 189 & 23 & - & 157 \\
\hline 6 & 222 & 11 & - & 187 \\
\hline 7 & 400 & 35 & + & 203 \\
\hline 8 & 218 & 42 & - & 57 \\
\hline 9 & 475 & 36 & - & 143 \\
\hline 10 & 212 & 59 & + & 154 \\
\hline 11 & 277 & 36 & - & 41 \\
\hline 12 & 153 & 16 & + & 176 \\
\hline 13 & 265 & 22 & - & 100 \\
\hline 14 & 96 & 6 & + & 39 \\
\hline 15 & 50 & 7 & - & 87 \\
\hline 16 & 408 & 52 & + & 125 \\
\hline 17 & 302 & 28 & + & 125 \\
\hline 18 & 99 & 17 & - & 47 \\
\hline 19 & 165 & 10 & + & 83 \\
\hline 20 & 267 & 21 & + & 83 \\
\hline 21 & 196 & 24 & - & 32 \\
\hline 22 & 182 & 19 & + & 69 \\
\hline 23 & 310 & 44 & + & 62 \\
\hline 24 & 387 & 36 & + & 34 \\
\hline 25 & 332 & 21 & + & 31 \\
\hline
\end{tabular}


insertion of the endoprosthesis, despite prophylactic antibiotic treatment in eight of them. Instillation or continuation of antibiotic treatment was successful in all 11 patients. No complications of the papillotomy were seen.

In two patients cholangitis developed respectively $3 \frac{1}{2}$ and five months after insertion of the endoprosthesis. In both patients the endoprosthesis had moved upwards above the stenosis. In one patient the endoprosthesis could be pulled back into position. In the other patient a second endoprosthesis was inserted. At present we use an endoprosthesis with two barbs to prevent dislodgement in both directions. In one patient recurrent jaundice developed because of clogging of the endoprosthesis six months after insertion. The endoprosthesis was removed and a new one inserted. Five additional patients had recurrent jaundice shortly before death. In one patient a cholecystitis occurred six months after insertion of the endoprosthesis because of cystic duct occlusion. Cholecystectomy was performed, but she died from postoperative complications.

No patient needed a gastroenterostomy because of duodenal obstruction.

\section{Discussion}

Curative resections of malignancies causing obstructive jaundice can be attempted in only a small minority of patients. In most other patients only palliative bypass procedures are possible. The mortality of these surgical bypass procedures, however, is high, even up to $33 \% .^{9}$ Non-surgical methods to decompress the biliary system are percutaneous transhepatic drainage and percutaneous introduction of an endoprosthesis. This percutaneous approach has several disadvantages together with a considerable complication rate, mainly because a long, rather large, puncture channel through the liver tissue is made. A biliary fistula often remains, when the permanent positioning of a percutaneous prosthesis fails. ${ }^{10-12}$

It was logical to expect that an endoscopic technique should be developed for introduction of biliary endoprostheses through the natural orifice of the papilla and the natural common duct channel, thereby avoiding the main disadvantage of the percutaneous approach. Up to the present it has only been possible to introduce $2.1 \mathrm{~mm}$ diameter endoprostheses and, in our experience, these endoprostheses do not always guarantee an unimpeded bile flow. With the techniques described here, it is possible to introduce larger $3.2 \mathrm{~mm}$ diameter endoprostheses via the transpapillary route. The results obtained in 45 patients show that the biliary flow through such endoprostheses was adequate in all patients.

Cholangitis and chills shortly after insertion of the endoprosthesis occurred in 11 patients and could easily be managed conservatively. To avoid this complication, it is imperative to sterilise all ancillary equipment and to properly disinfect the endoscopes. For this purpose a special washing disinfecting machine was developed, capable of disinfecting also the minute channels supplying air and water within the endoscope. ${ }^{13}$ Using this machine no instance of iatrogenic infection within the bile duct has been observed. Therefore routine prophylactic antibiotics were omitted.

Late cholangitis occurred only in case of dislodgement or clogging of the endoprosthesis. Ascending cholangitis probably does not occur when the endoprosthesis is patent.

We prefer to position the endoprosthesis projecting from the papilla into the duodenum. This facilitates removal of the endoprosthesis if clogging occurs. When the lower end of the prosthesis is within the common duct it is nearly impossible to grasp and remove it. There is some fear that a transpapillary prosthesis may cause compression of the pancreatic orifice with subsequent pancreatitis. We have never observed pancreatitis in our patients. Whether this is explained by the routine papillotomy associated with the insertion of the prosthesis is uncertain at present, although this explanation seems to be plausible. No patients had complaints suggestive of injuries to the duodenal wall by the endoprosthesis because of pressure necrosis. Nor were injuries of the duodenal wall ever seen in those patients who had an endoscopy later on.

The endoscopic positioning of a biliary endoprosthesis has become a relatively easy procedure for experienced endoscopists since the introduction of a duodenoscope with a $3.7 \mathrm{~mm}$ biopsy channel. In two situations, however, the procedure remains very difficult or even impossible. In a very low bile duct stenosis it may be difficult to perform an adequate papillotomy, because proper introduction of the papillotome is prohibited. Tumours of the bifurcation can usually be passed with a flexible atraumatic guide wire entering both lobes of the liver. Introduction, however, of a catheter over this guidewire sometimes fails because the tumorous stenosis is too scirrhous to allow passage of the thicker Teflon catheter. Dilatation devices should be developed to overcome these difficulties, because optimal drainage is only achieved when a catheter can be positioned in both lobes of the liver. At present often only one lobe can be drained.

Many of the late complications related to biliary 
endoprostheses are due to clogging. Experiments are necessary to find out the most suitable material for the endoprostheses to avoid or retard this clogging phenomenon. At present it is not clear whether choleretic drugs or gallstone-dissolving agents are helpful in avoiding this clogging.

The first experiences with endoscopically positioned $3.2 \mathrm{~mm}$ biliary endoprostheses are promising and suggest that this method may well become a great challenge to the percutaneous approach and possibly in the near future for the surgical bypass procedure as well. It is our conviction at present that the transpapillary approach should be the method of choice for all patients with malignant obstructive jaundice, who are at increased surgical risk. Whether this approach should be extended to all patients with malignant jaundice will only be known after properly controlled trials studying mortality, morbidity, survival time, and cost effectiveness together with the overall efficacy of drainage.

\section{References}

1 Cotton PB. ERCP Progress report. Gut 1977; 18: 316-41.

2 Zimmon DS, Chang J, Clemett AR. Advances in the management of bile duct obstruction. Med Clin North Am 1979; 63: 539-609.

3 Cotton PB, Burney PGJ, Mason RR. Transnasal bile duct catheterisation after endoscopic sphincterotomy. Gut 1979; 20: 285-7.

4 Wurbs D, Phillip J, Classen M. Experiences with the long standing nasobiliary tube in biliary diseases. Endoscopy 1980; 12: 219-23.

5 Soehendra N, Reijnders-Frederix V. Palliative bile duct drainage. A new endoscopic method of introducing a transpapillary drain. Endoscopy 1980; 12: 8-11.

6 Lawrence BH, Cotton PB. Decompression of malignant biliary obstruction by duodenoscope intubation of bile duct. $\mathrm{Br}$ Med J 1980; 280: 522-3.

7 Huibregtse K, Haverkamp HJ, Tytgat GN. Transpapillary positioning of a large $3.2 \mathrm{~mm}$ biliary endoprosthesis. Endoscopy 1981; 13: 217-9.

8 Huibregtse K. New developments in gastro-intestinal endoscopy. In: Proceedings of 'geriatrics for the practitioner'. Amsterdam: Excerpta Medica, 1981: 67-74.

9 Feduska NJ, Dent T, Lindenauer SM. Results of palliative operations for carcinoma of the pancreas. Arch Surg 1971; 103: 330-4.

10 Hoevels J, Lunderguist A, Ihse J. Percutaneous transhepatic intubation of bile ducts for combined internalexternal drainage in preoperative and palliative treatment of obstructive jaundice. Gastroint Rad 1978; 3: 23-31.

11 Burcharth $F$, Ingemann Jensen L, Olesen $\mathrm{K}$. Endoprosthesis for internal drainage of the biliary tract. Gastroenterology 1979; 77: 133-7.

12 Tytgat GN. Endoscopic methods of treatment of gastrointestinal and biliary stenoses. Endoscopy 1980; suppl: 57-68.

13 Huibregtse K, Haverkamp $\mathrm{HJ}$, Hoogland $\mathrm{MN}$, et al. A new instrument for cleaning and disinfection of fiberendoscopes. Acta Endosc 1981; 11: 363-7. 